\title{
PERFORMANCE EVALUATION OF TCP SACK1 IN WIMAX NETWORK ASYMMETRY
}

\author{
Kailash Chandra Bandhu', Rajeev G. Vishwakarma ${ }^{2}$ \\ ${ }^{1}$ Ph.D. Scholar, Department of Computer Science \& Engineering, Bhagwant University, Ajmer, India \\ ${ }^{2}$ Professor, Department of Computer Science \& Engineering, Shri Vaishnav Institute of Technology and Science, \\ Indore, India
}

\begin{abstract}
The WiMAX technology support to different channel bandwidth, cyclic prefix, modulation coding scheme, frame duration, simultaneous two way data transfer and propagation model. The WiMAX network asymmetry is largely depends on DL: UL ratio. This paper evaluate the performance of TCP Sackl by considering Channel Bandwidth, Cyclic Prefix, Modulation Coding Scheme, Frame Duration, Two way transfer and Propagation model in WiMAX network with network asymmetry. The performance of TCP Sack1 in WiMAX network is evaluating by varying MAC layer parameter such as channel bandwidth, cyclic prefix, modulation coding scheme, frame duration, DL: UL ratio and physical layer parameter such as propagation model and full duplex mode of data transfer and other operating parameter such as downloading traffic and these parameters really affect the performance of TCP Sack1 in WiMAX network.
\end{abstract}

The performance of WiMAX network is measured in terms of throughput, goodput and number of packets dropped.

Keywords: World Wide interoperability for microwave access (WiMAX), Subscriber Stations (SSs), Downlink (DL), Uplink (UL), Medium access control (MAC), Transmission Control Protocol (TCP), OFDM, IEEE 802.16, Throughput, Goodput and Packets drop

\section{INTRODUCTION}

WiMAX is abbreviation 'Worldwide Interoperability for Microwave Access', is a new wireless OFDM based technology that provides high throughput broadband connection over long distances based on IEEE 802.16 [8][9].

WiMAX network increasingly more intelligent and agile communication systems, capable of providing spectrally efficient and flexible data rate access [9]. Network asymmetry means that network characteristics in one direction do not match with the other direction [13].

The channel bandwidth, cyclic prefix, modulation coding scheme, frame duration, simultaneous two way data transfer and propagation model affect TCP performance along with Network asymmetry, since TCP relies on the timely arrival of acknowledgments (ACKs) to increase its congestion window and data sending rate. Under normal network conditions, an ACK is duly received for packets sent, and this helps the sender to increase the data sending rate. In the case of congestion, typically indicated by packet loss, TCP abruptly decreases its congestion window, and retransmits the lost packets [10][11]. The retransmission may aggravate the congestion. Normally, there are two ways to indicate the packet loss or congestion:

(1) Expiry of retransmission timer (2) Receipt of three or more duplicate ACKs
In the presence of an imperfect ACK channel, the ACK clocking is disrupted, i.e., packets sent are not duly acknowledged. Consequently, at the sender, the timer expires which TCP interprets as congestion, the congestion window plummets and the packets are retransmitted, even though these packets may have correctly reached to the receiver. This implies that the TCP throughput and goodput not only depends on the characteristics of the data sending channel, but also on the reverse channel used by ACKs [13][7][3].

TCP with 'Selective Acknowledgments' is an extension of Reno and it works around the problems faced by Reno and New Reno namely detection of multiple lost packets and retransmission of more than one lost packet per RTT [14].

Sack retains the slow start and fast retransmits parts of Reno. It also has the coarse grained timeout of Tahoe to fall back on, in case a packet loss is not detected by the modified algorithm.

Sack requires that segments not be acknowledged cumulatively but should be acknowledged selectively. Thus each ACK has a block which describes which segments are being acknowledged. Thus the sender has a picture of which segments have been acknowledged and which are still outstanding [14]. 
Whenever the sender enters fast recovery, it initializes a variable pipe which is an estimate of how much data is outstanding in the network, and it also set congestion window (cwnd) to half the current size. Every time it receives an ACK it reduces the pipe by 1 and every time it retransmits a segment it increments it by 1 [14].

Whenever the pipe goes smaller than the congestion window (cwnd) window it checks which segments are not received and send them. If there are no such segments outstanding then it sends a new packet [14]. Thus more than one lost segment can be sent in one RTT.

The biggest problem with Sack is that currently selective acknowledgements are not provided by the receiver, to implement Sack it need to implement selective acknowledgment which is not a very easy task [14].

WiMAX provides the flexibility in channel bandwidth [2], cyclic prefix, modulation coding scheme, frame duration, propagation model and simultaneous two way data transfer [1].

The rest of the paper is structured as follows. The system model for the investigation is introduced in Section 2. In Section 3, simulation scenarios are presented and the results are discussed. Finally, the conclusion is present in Section 4.

\section{NETWORK MODEL}

This section present the network model used in investigation. The network topology is shown in Figure 1. All subscriber stations are downloading stations (SSs).

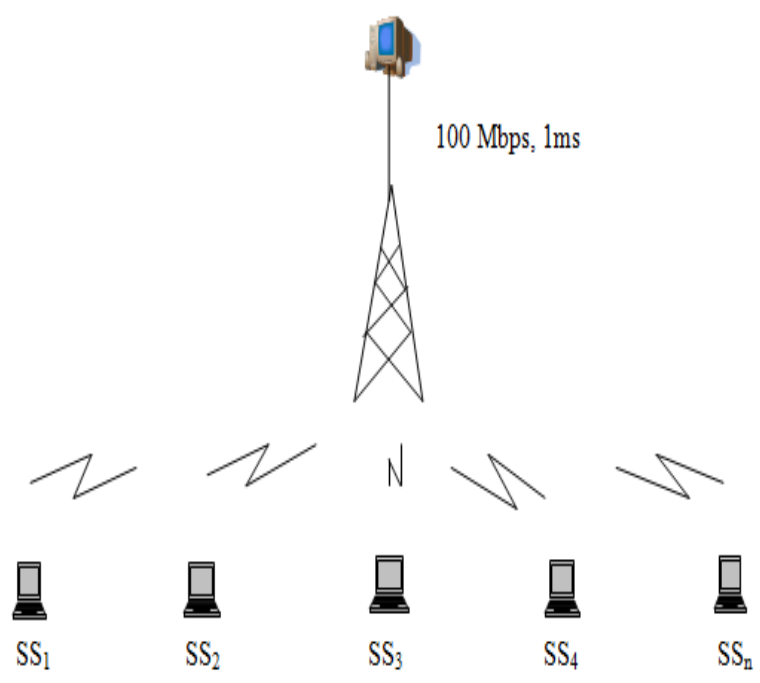

Fig -1: Network Topology

\subsection{Simulation Environment}

The performance analyzed through simulation by using network simulator ns-2 (ns2.31) and the WiMAX module from the National Institute of Standards and Technology (NIST) [3][4].
The simulation parameters are summarized in Table 1.

Table -1: Simulation Parameters

\begin{tabular}{|l|l|}
\hline \multicolumn{2}{|l|}{ WiMAX and OFDM Parameters } \\
\hline Channel bandwidth & $3,7,10,20,28 \mathrm{MHz}$ \\
Frame duration & $5,10 \mathrm{~ms}$ \\
Modulation \& Coding & BPSK 1/2, QPSK 1/2, \\
& QPSK 3/4, 16QAM 1/2, \\
& 16QAM 3/4, 64QAM \\
Cyclic prefix & 2/3,64QAM 3/4 \\
Contention size & 1/2, 1/4, 1/8, 1/16 \\
Propagation Model & 5 \\
& TworayGround, \\
& FreeSpace, Shadowing \\
\hline Traffic Source and Other Parameters \\
\hline TCP version & Sack1 \\
TCP segment size & 960 Bytes \\
No. of DL WL Nodes & $5,10,15,20,25$ \\
Delayed ACK factor & $2 \mathrm{~s}$ \\
TCP start time & $20 \mathrm{~s}$ \\
Simulation duration & $300 \mathrm{~s}$ \\
\hline
\end{tabular}

\subsection{Performance Metrics}

The performance studied by means of three metrics:

1) Throughput: that measures the amount of raw bytes sent by a source.

2) Goodput: that measures bytes that are sent and success-fully acknowledged.

3) Number of Packets drop.

\section{SIMULATION RESULTS}

Several scenarios are considered to analyze the performance of TCP Sack1 in WiMAX network for different DL: UL ratio.

\subsection{Scenario 1: Effect of Channel Bandwidth}

The figure 2 proved that larger channel bandwidth and larger DL:UL ratio provides higher throughput and goodput and lower channel bandwidth and lower DL:UL ratio provides lower throughput and goodput in WiMAX network. Because higher DL:UL ratio and higher bandwidth support to large downloading traffic. The channel bandwidth $28 \mathrm{MHz}$ has maximum throughput and goodput for DL:UL ratio 0.75 .

The figure 3 shows the number of packets dropped for different channel bandwidth with different DL:UL ratio. When the channel bandwidth increased the number of packets dropped also increases for different DL:UL ratio.

The large number of packets dropped for larger channel bandwidth and larger DL:UL ratio. Less number of packets dropped for lower channel bandwidth and lower DL:UL. Because wireless channel is unreliable channel and higher channel bandwidth and higher DL:UL ratio carry the large traffic in downloading then the large number packets will be dropped. 
The channel bandwidth $28 \mathrm{MHz}$ has maximum number of packets dropped for DL:UL ratio 0.75.

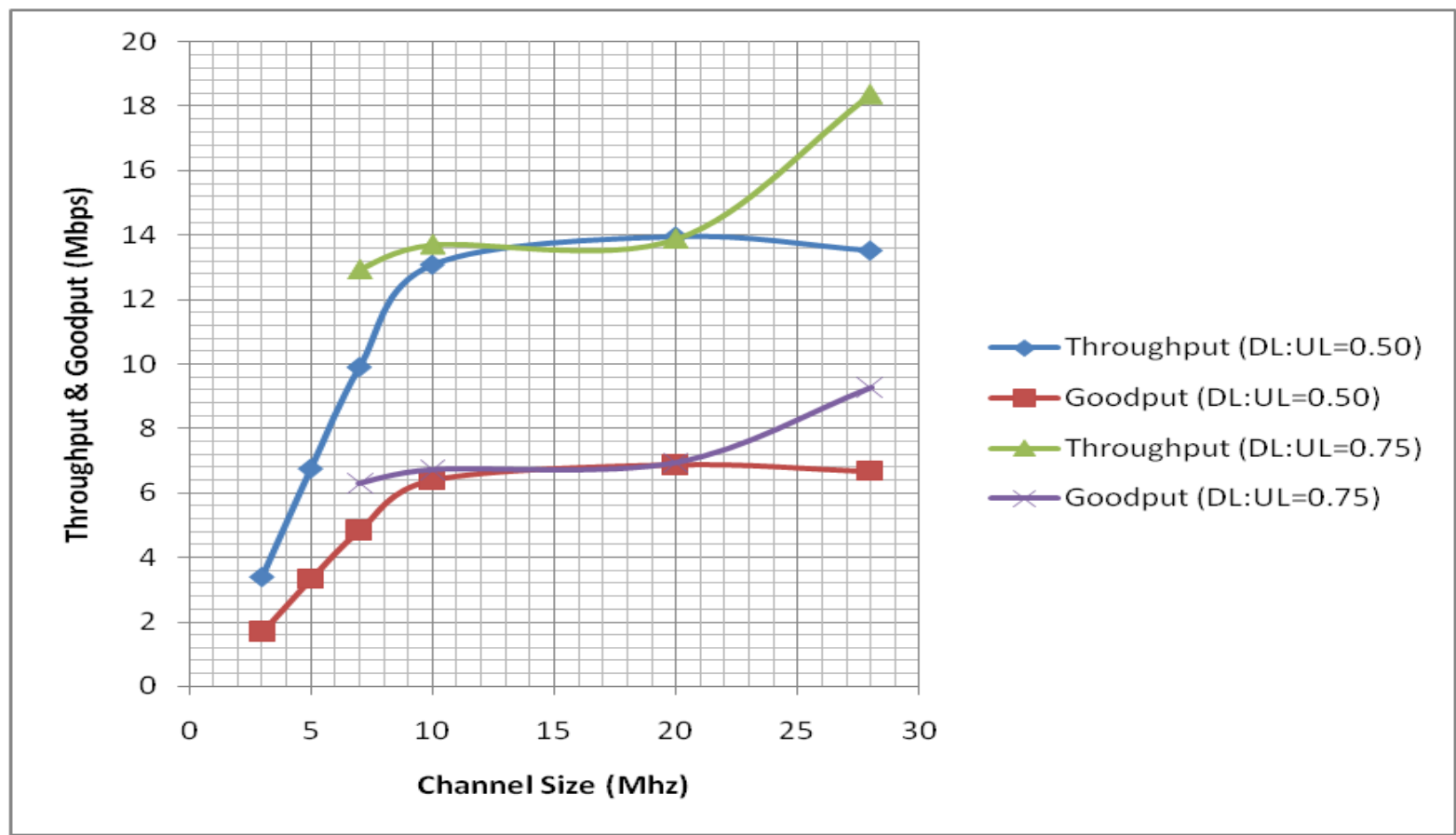

Fig -2: Throughput \& Goodput for different Channel Bandwidth with different DL:UL ratio

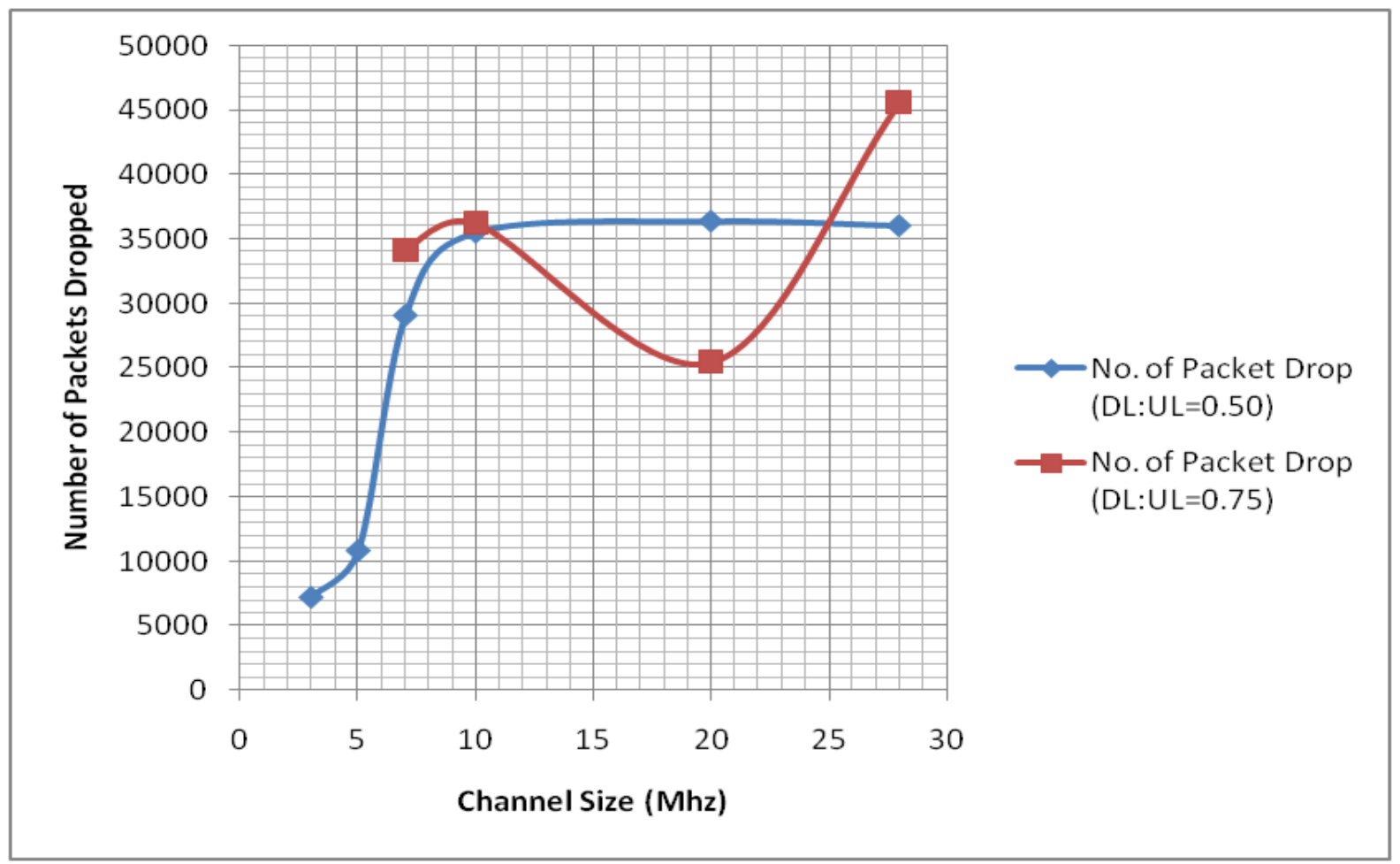

Fig -3: Number of Packets Dropped for different Channel Bandwidth with different DL: UL ratio

\subsection{Scenario 2: Effect Cyclic Prefix}

In this scenario the performance of TCP Sack1 in WiMAX network is analyzed using cyclic prefix in bandwidth asymmetry, shown in figure 4 and 5 .

Figure 4 shows that, whenever the cyclic prefix increases the throughput and goodput decreases. The higher cyclic prefix has low throughput and goodput due to extra overhead of cyclic prefix and lower cyclic prefix has higher throughput and goodput. 
The maximum throughput and goodput obtained for cyclic prefix 0.3125 with DL:UL ratio 0.90 .

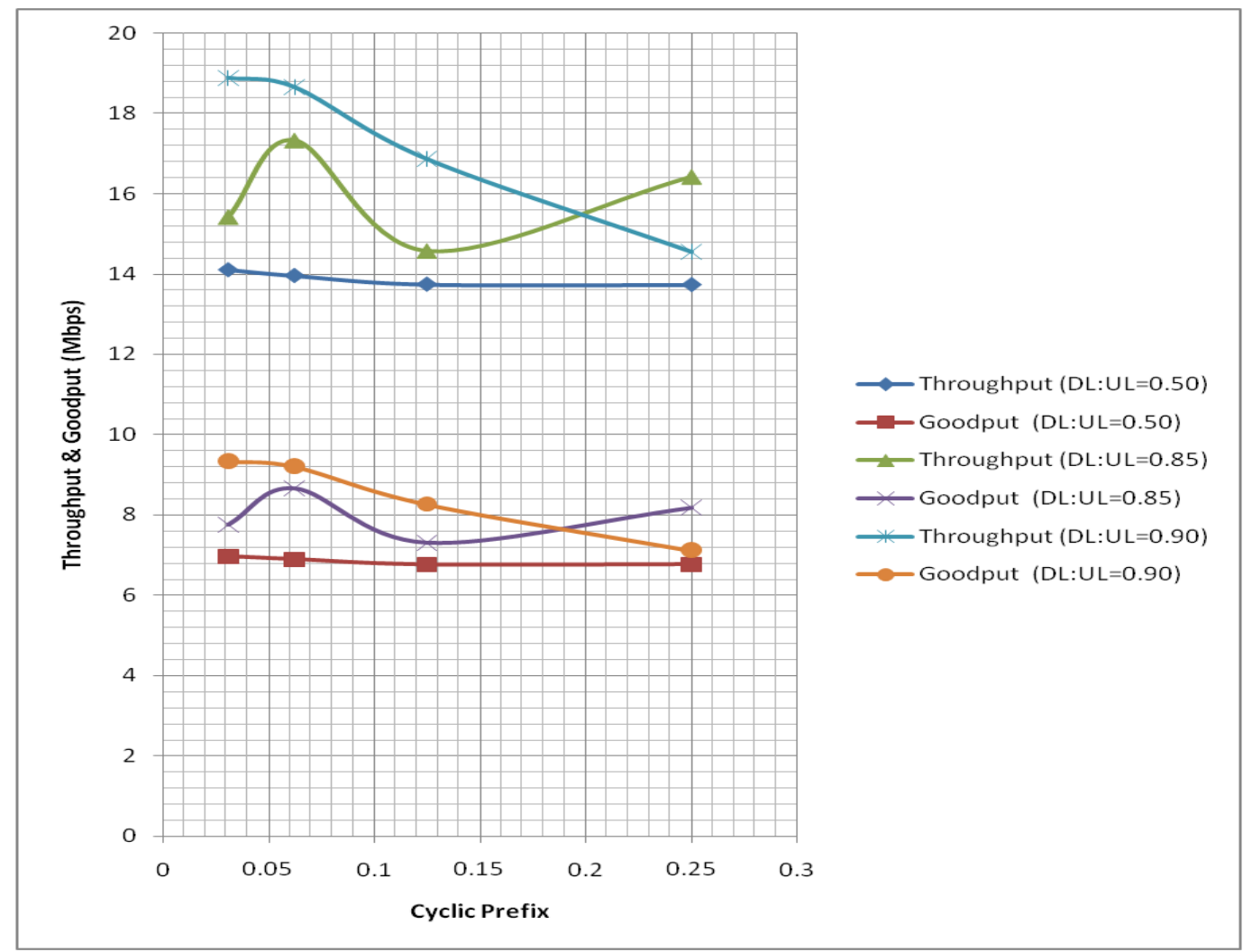

Fig -4: Throughput \& Goodput for different Cyclic Prefix by considering DL:UL ratio

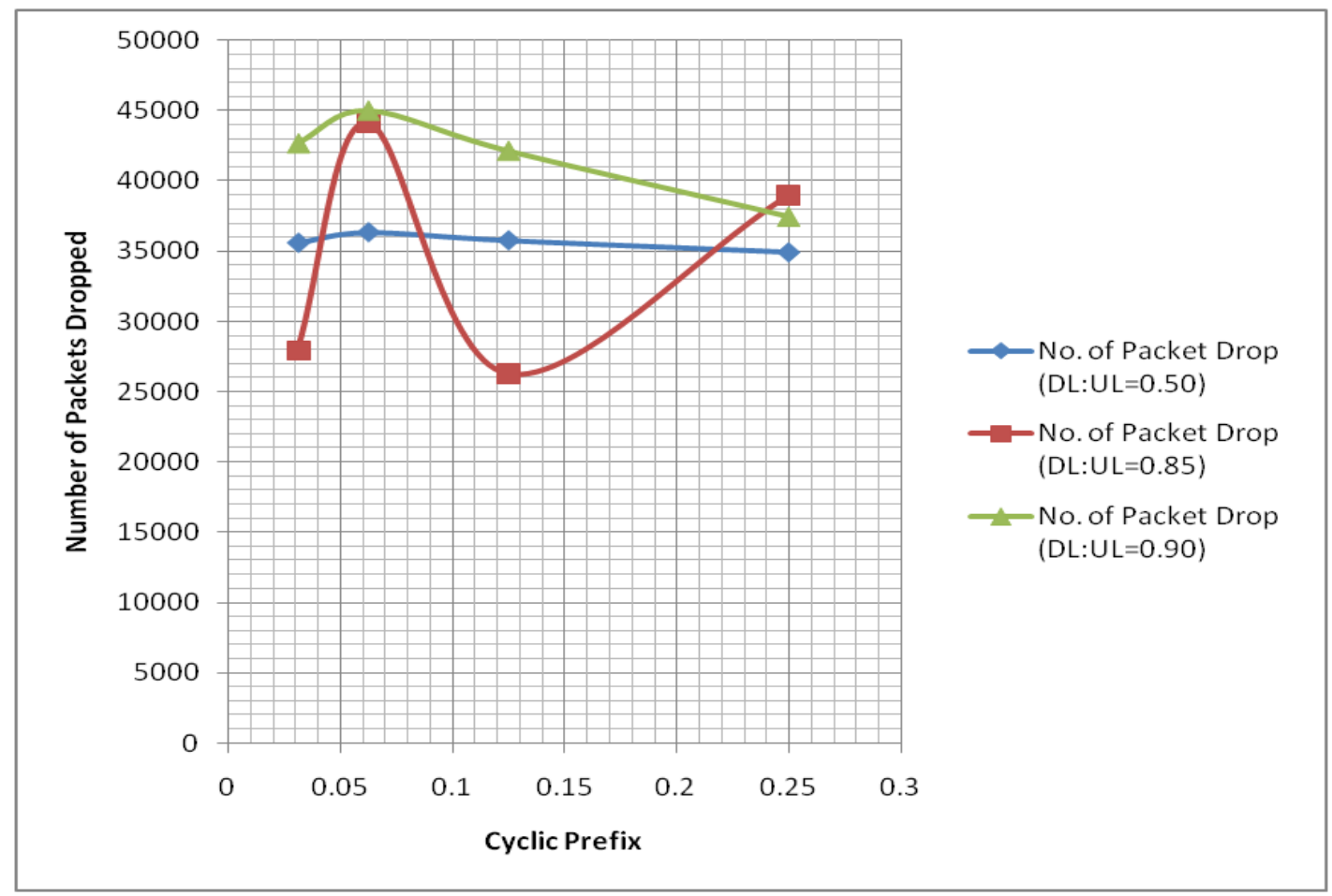

Fig -5: No. of Packet drop for different Cyclic Prefix by considering DL:UL ratio

The number of packets drop is higher for low cyclic prefix due to echo and interference in multipath propagation. The low cyclic prefix increases the echo and interference in multipath propagation due to this packet drop increases which is shown in figure 5.

The maximum number packet drop obtained for cyclic prefix 0.3125 with DL:UL ratio 0.90 . 


\subsection{Scenario 3: Effect of Modulation Coding Scheme}

The figure 6 shows the throughput, goodput and figure 7 shows the number of packets drop in different modulation coding scheme for different DL:UL ratio.

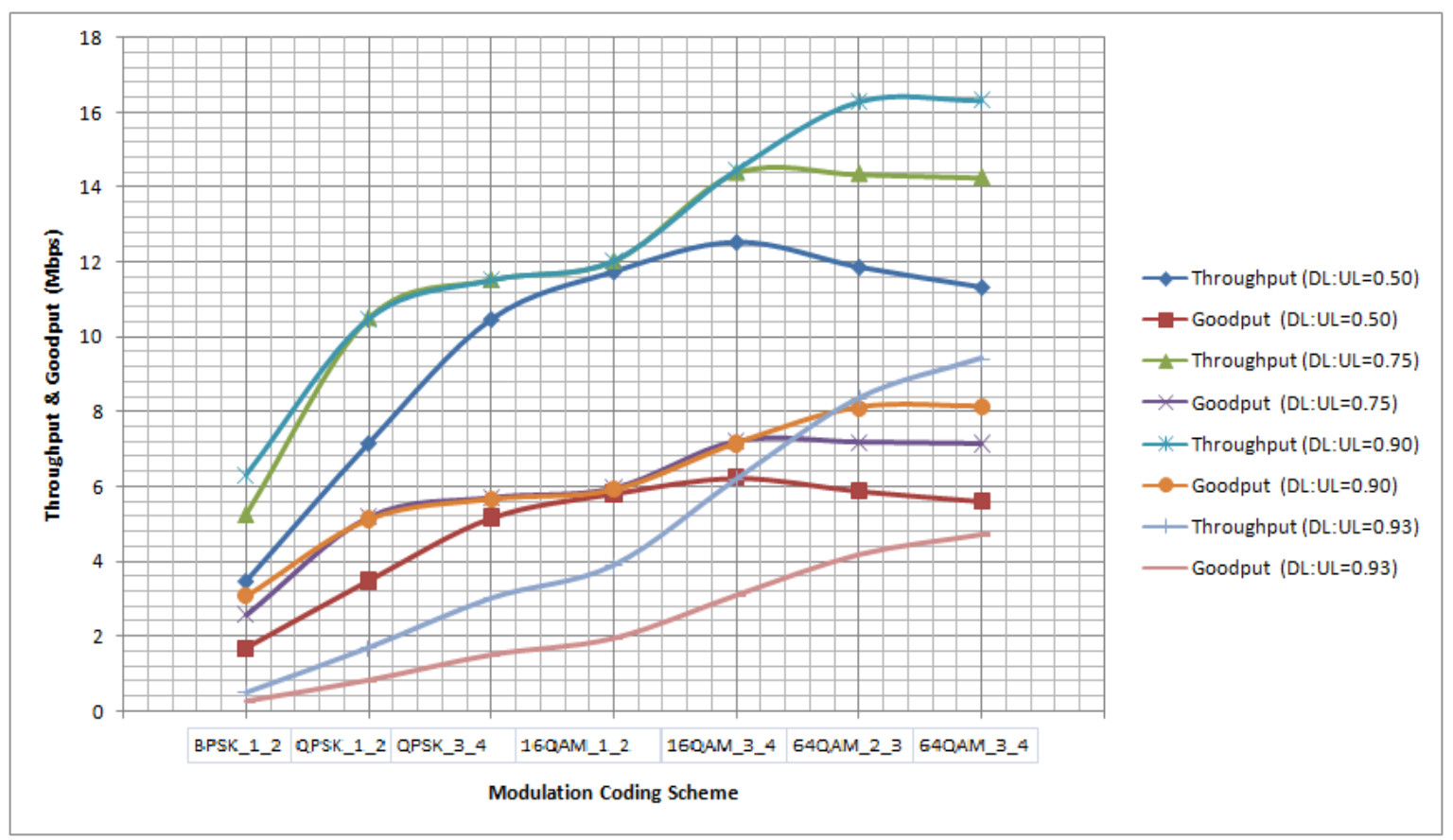

Fig -6: Throughput \& Goodput for different Modulation Coding Scheme by considering DL:UL ratio

The throughput and goodput for DL:UL ratio 0.90 is higher as compare to other DL:UL ratio for modulation coding scheme 64QAM_3_4 due to large number of time slots in higher order modulation coding scheme as compare to lower order modulation coding scheme shown in figure 6. Higher order modulation coding scheme support higher data rate.

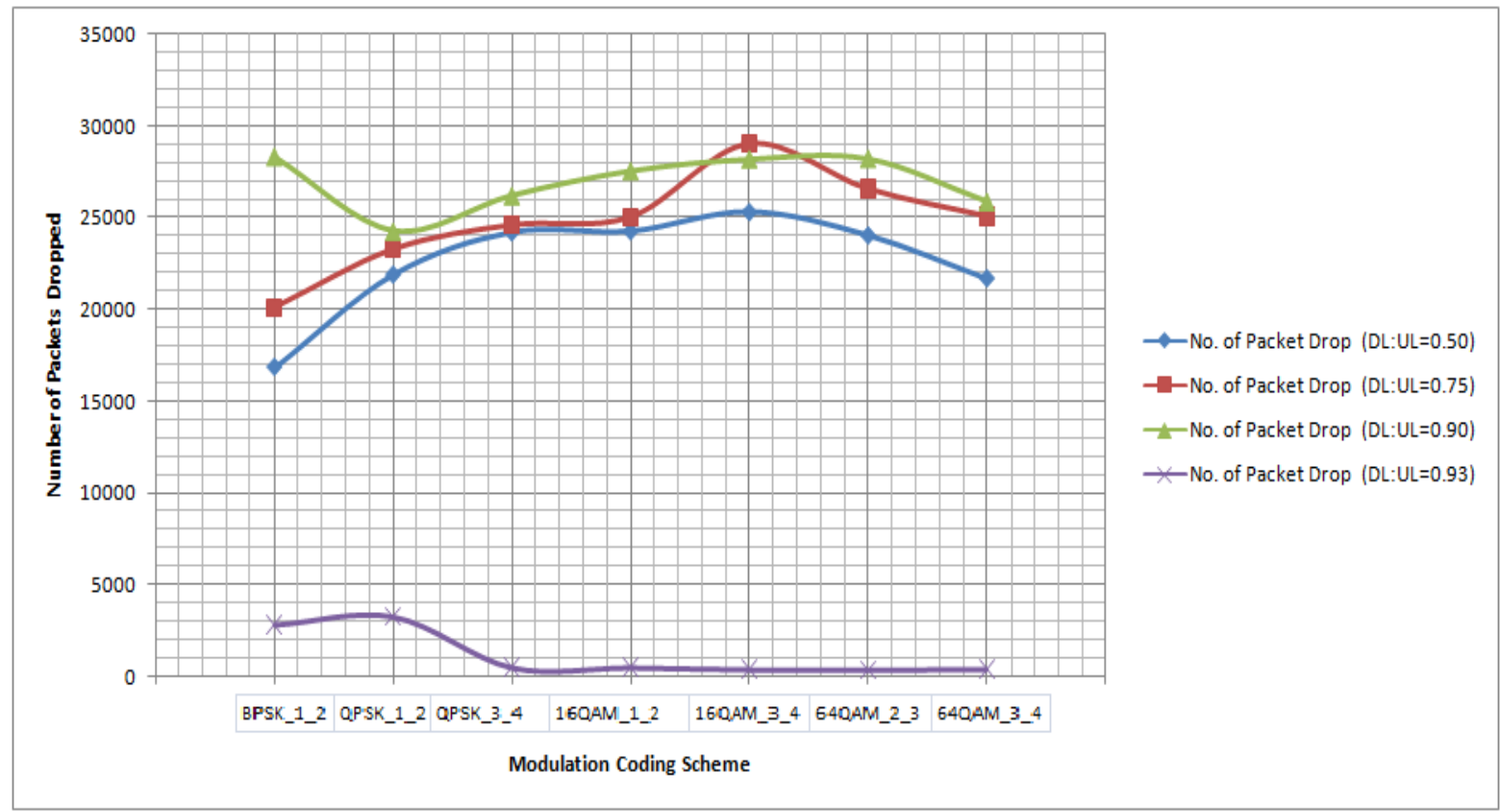

Fig -7: No. of Packet drop for different Modulation Coding Scheme by considering DL:UL ratio

The number of packets drop is higher for DL:UL ratio 0.90 with 64QAM_3_4.

The number of packets drop increases because the higher order modulation coding scheme support to high data rate but signal become poor due to long distance so that the start packet drop which shown in figure 7. 


\subsection{Scenario 4: Effect of Frame Duration}

The figure 8 represents the effect of frame duaration in terms of throughput and goodput and figure 9 shows effect in number of packets drop.

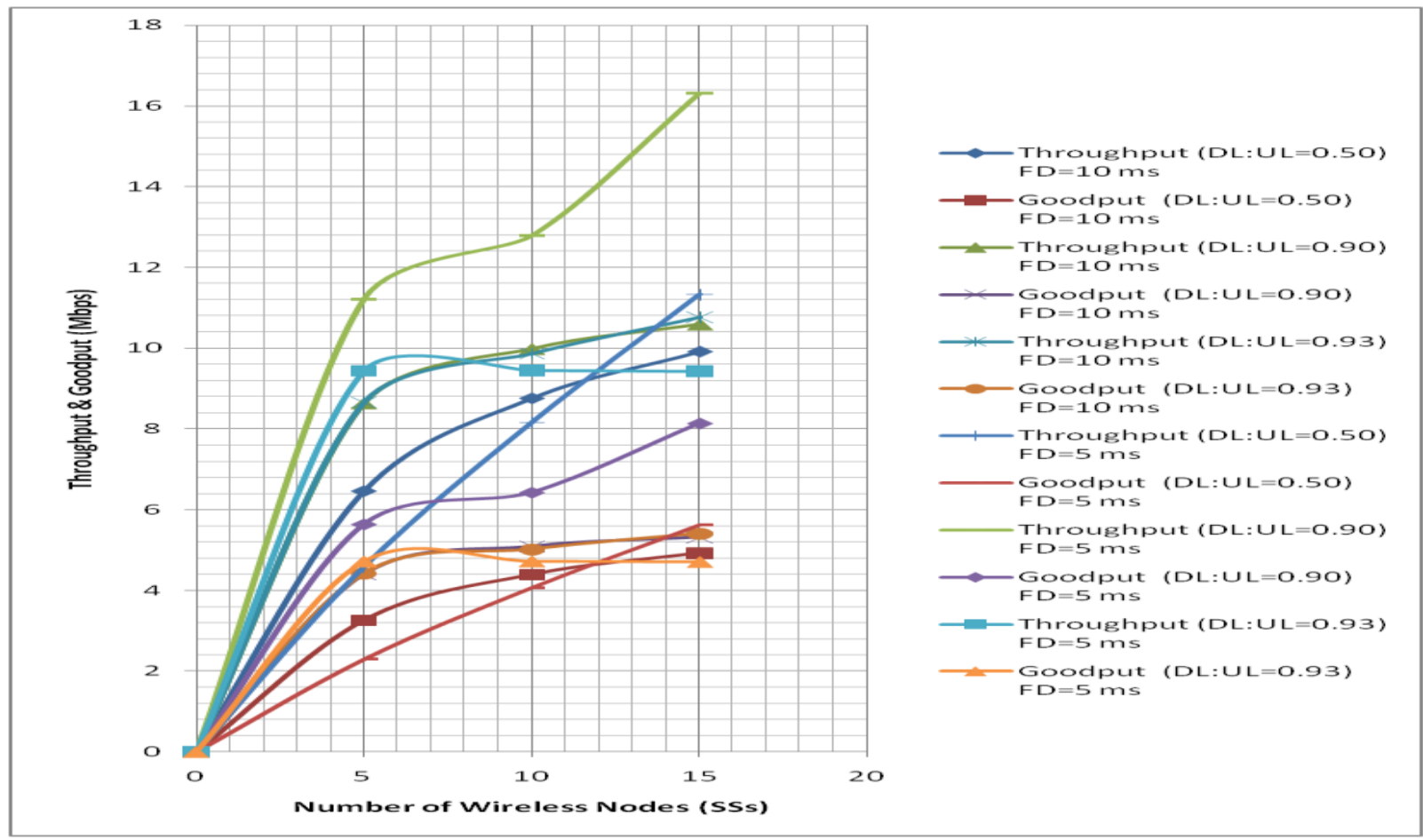

Fig -8: Throughput \& Goodput for different Frame Duration by considering DL:UL ratio

The figure 8 shows that the throughput and goodput for DL:UL ratio 0.90 with frame duration $5 \mathrm{~ms}$ is higher as compare to $10 \mathrm{~ms}$. When Frame duration increases the throughput and goodput decreases due to the less number of frame present in channel per unit time.

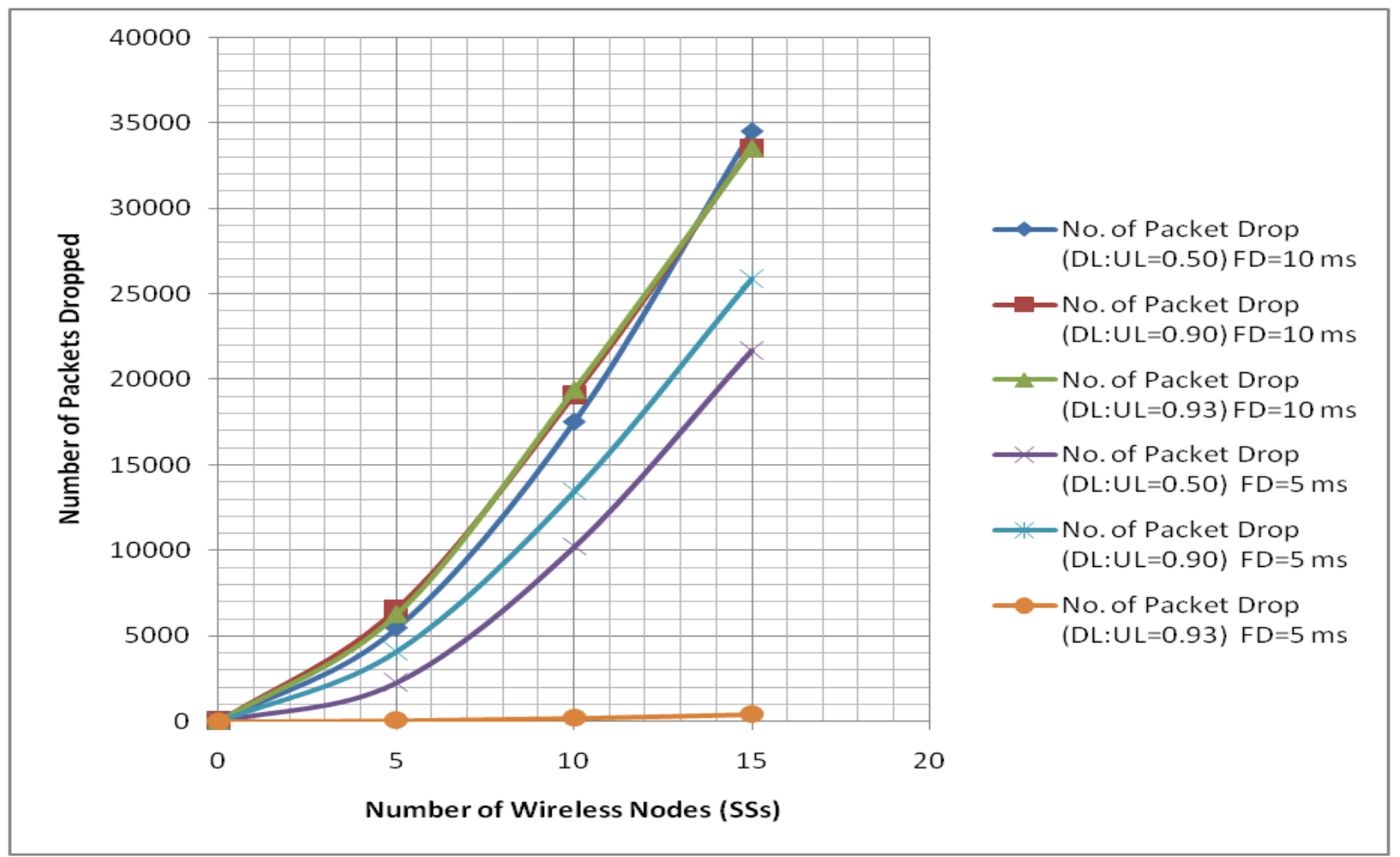

Fig -9: No. of Packet drop for different Frame Duration by considering DL:UL ratio 
The throughput and goodput for DL:UL ratio 0.93 is less for frame duration $5 \mathrm{~ms}$ and 10ms as compare to DL:UL ratio 0.90 for $5 \mathrm{~ms}$ and $10 \mathrm{~ms}$ due to greater bandwidth asymmetry. If DL:UL ratio is 0.50 then there is no bandwidth asymmetry.

The number of packets drop is larger for DL:UL ratio 0.50 with $10 \mathrm{~ms}$ frame duration, shown in figure 9.

\subsection{Scenario 5: Effect of Simultaneous Two Way Transfer}

Figure 10 and 11 shows the effect of simultaneous two way data transfer.

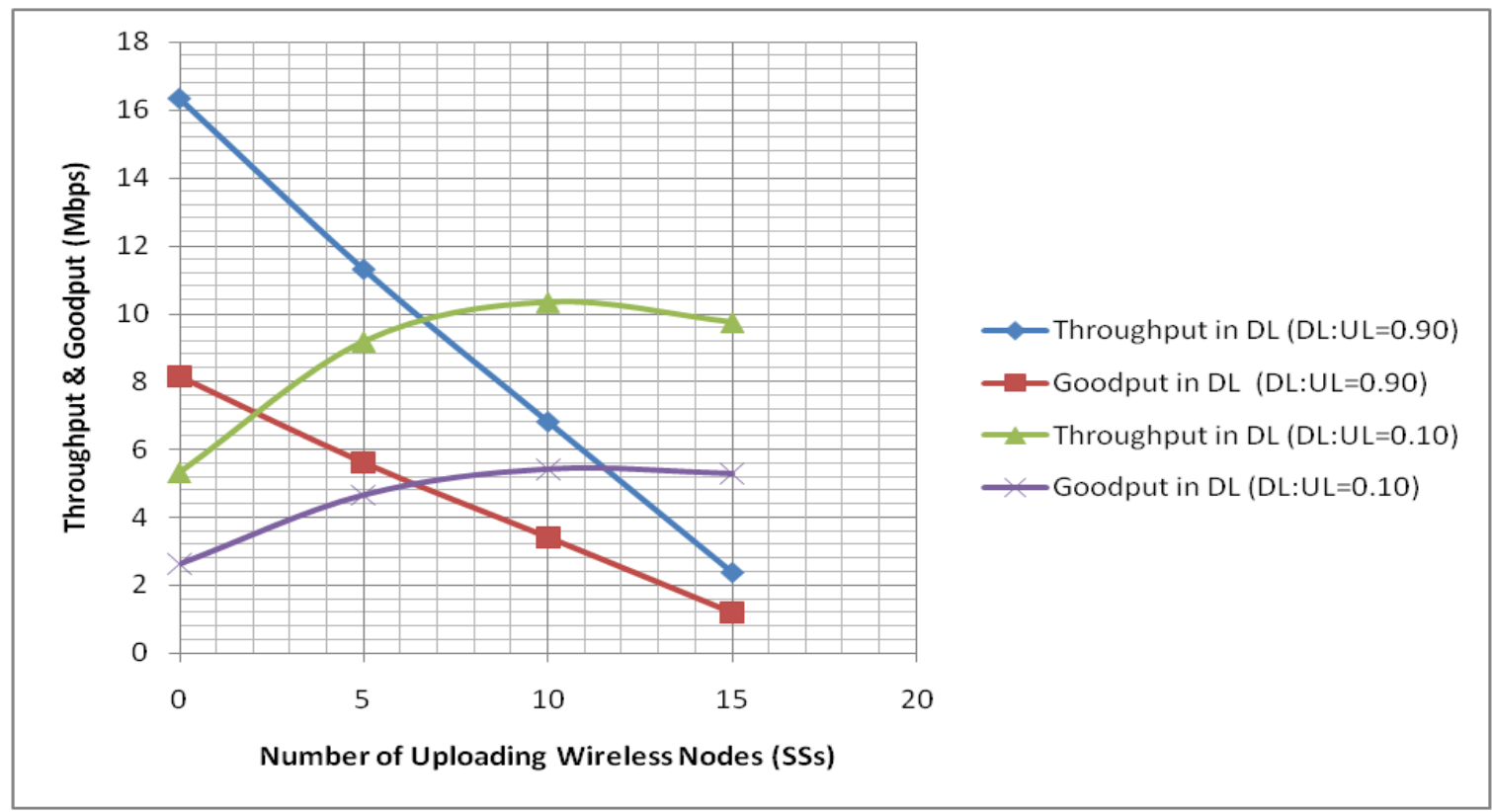

Fig -10: Throughput \& Goodput for Simultaneous two way transfer by considering DL:UL ratio

The figure 10 represent the throughput and goodput for DL:UL ratio 0.90 and 0.10 . Whenever the number of uploading nodes increases the throughput and goodput goes down due to portion alloted to UL is 0.10 (DL:UL=0.90) which is not sufficient to handel large number of uploading nodes.

Similarly the throughput and goodput increases when the number of uplaoding nodes increases due to portion allotted to UL is 0.90 (DL:UL=0.10) which is capable handle large uploading traffic.

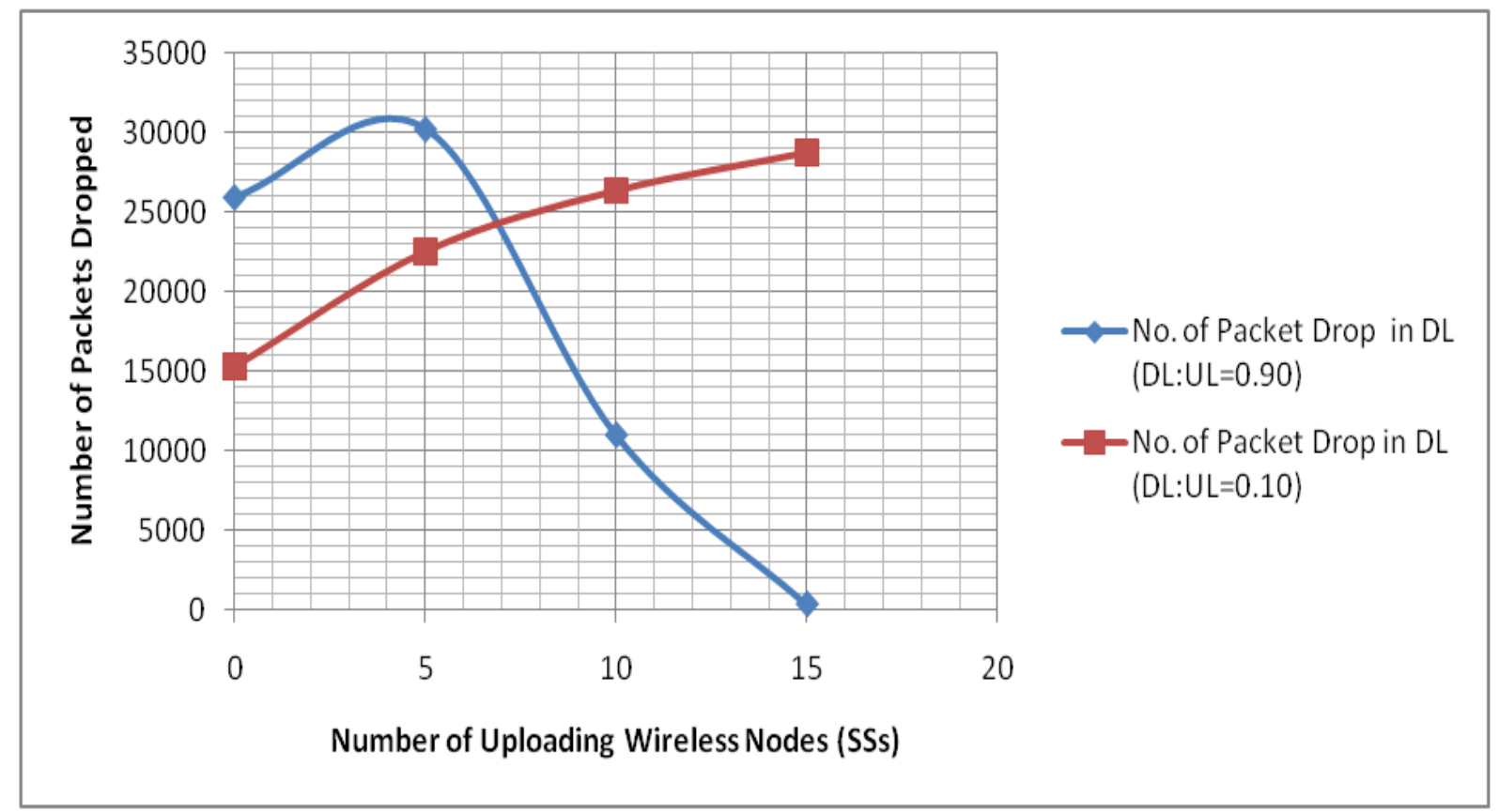

Fig -11: No. of Packet drop for Simultaneous two way transfer by considering DL:UL ratio 
The number of packet drop increases for DL:UL ratio $0.10(\mathrm{UL}=0.90)$ due to large traffic in uploading and number of packet drop decreases when DL: $\mathrm{UL}$ ratio $0.90(\mathrm{UL}=0.10)$ due to low traffic in uploading shown in figure 11.

\subsection{Scenario 6: Effect of Propagation Model}

The impact of propagation model is measured by three performance matrices throughput, goodput and number of packets drop shown in figure 12 and 13.

The throughput and goodput are same for all propagation model for DL:UL ratio 0.90, shown in figure 12.

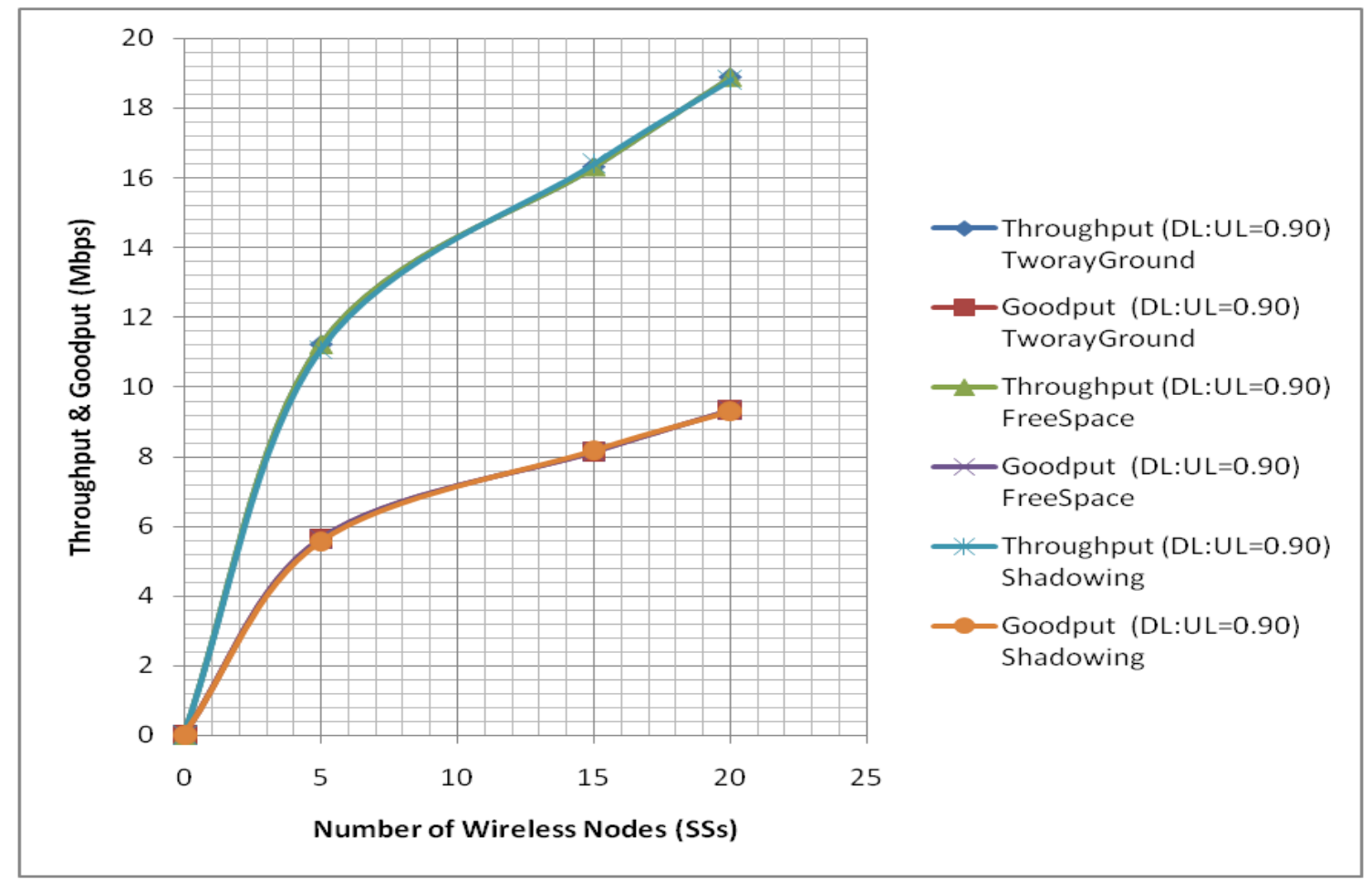

Fig -12: Throughput \& Goodput for different Propagation model by considering DL:UL ratio

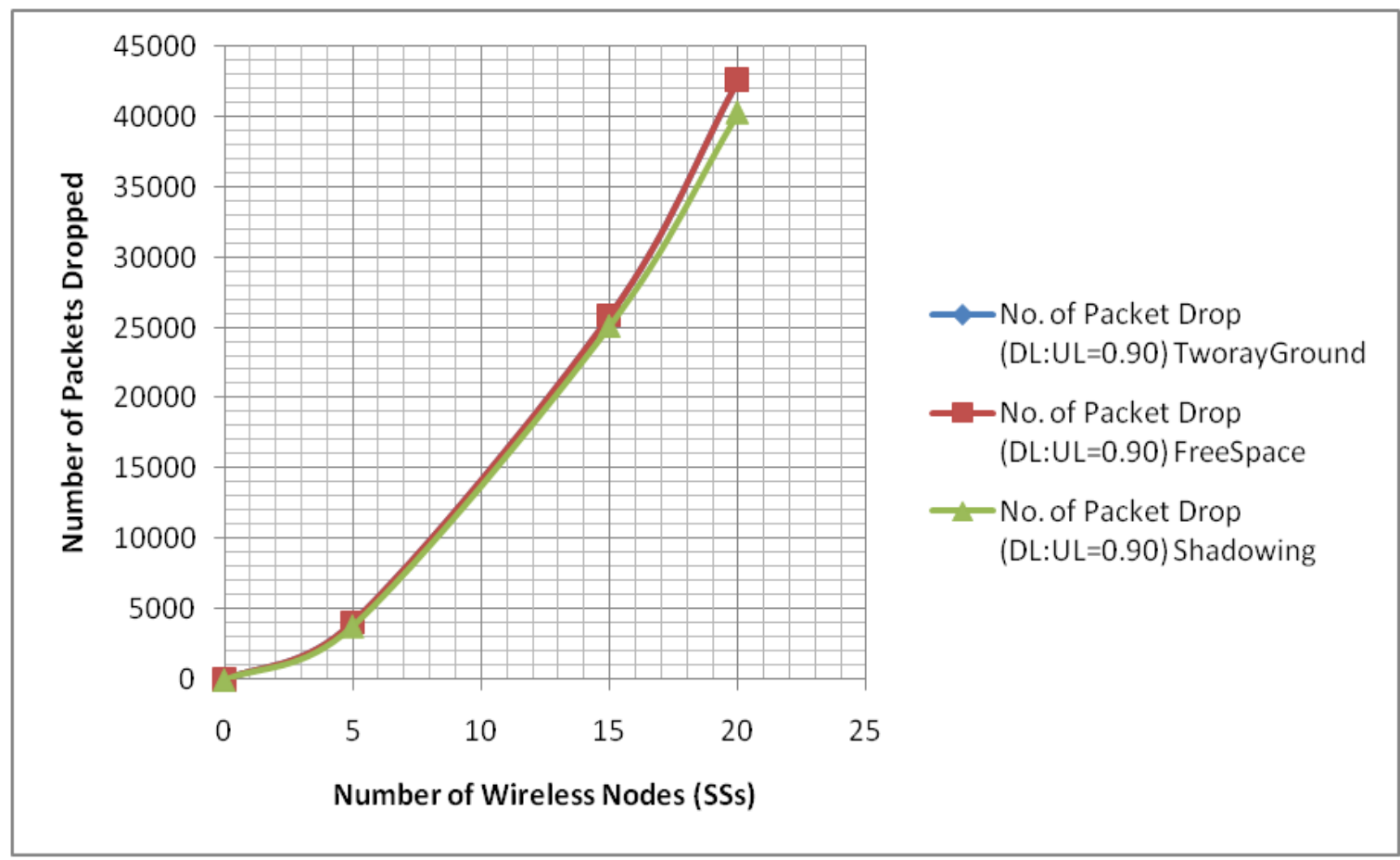

Fig -13: No. of Packet drop for different Propagation model by considering DL:UL ratio 
The number of packet drop is higher with DL:UL ratio 0.90 for TworayGround and FreeSpace propagation model due to reflection and refraction and this will be reason for weak signal strength, which is shown in figure 13 . The Shadowing has less number of packets dropped.

\section{CONCLUSION}

This paper analyzed the performance of channel bandwidth, cyclic prefix, modulation coding scheme, frame duration, simultaneous two way data transfer and propagation model, in WiMAX network with bandwidth asymmetry using TCP Sack1.

It is observed in simulation study that the higher channel bandwidth increases the data rate and number of packet dropped.

It is observed in simulation study that the higher cyclic prefix reduce the data rate also reduce the packet drop.

The higher order modulation coding scheme provides higher data rate and higher packet drop.

It is also observed that lower frame duration enhance the data rate and number of packet drop.

In simultaneous two way data transfer higher DL:UL ratio reduce data rate and packet drop for large uploading nodes and lower DL:UL ratio increase the data rate and packet drop for large uploading nodes.

The all propagation model provide same data rate and shadowing propagation model provide less number of packets dropped.

\section{REFERENCES}

[1]. Kailash Chandra Bandhu, Rajeev G. Vishwakarma "The Impact of Cyclic Prefix, Modulation Coding Scheme, Frame Duration, Two Way Transfer And Propagation Model With Network Asymmetry In WiMAX Network using TCP New Reno" International Journal of Engineering Research \& Technology (IJERT) ISSN: 2278-0181 Vol. 3 Issue 3, March - 2014

[2]. Kailash Chandra Bandhu, Rajeev G. Vishwakarma "The Impact of Channel Bandwidth with Network Asymmetry in WiMAX Network Using TCP New Reno" International Journal of Scientific \& Engineering Research Volume 4 (IJSER), Issue 2, February-2013, ISSN 2229-5518.

[3]. Konstantinos Tsiknas and George Stamatelos "Comparative Performance Evaluation of TCP Variants in WiMAX (and WLANs) Network Configurations" Hindawi Publishing Corporation Journal of Computer Networks and Communications Volume 2012, Article ID 806272, 9 pages doi:10.1155/2012/806272.

[4]. Pratyush Sharma, Abhishek Sharma, Kailash C. Bandhu "Performance Evaluation of Adaptive
Modulation Techniques and Offered Load in OFDM based WiMAX Network by Considering Cyclic Prefix" International Journal of Scientific \& Engineering Research Volume 2, Issue 11, November-2011 ISSN 2229-5518.

[5]. Network Simulator Ns-2, 2010, http://www.isi.edu/nsnam/ns.

[6]. R. Rouil, "The NIST WiMAX Network Simulator," NIST, Tech. Rep., 2007.

[7]. K. Xu, Y. Tian, and N. Ansari, "Improving TCP performance in integrated wireless communications networks," Computer Networks, vol. 47, no. 2, pp. 219-237, 2005.

[8]. IEEE Standard for Local and Metropolitan Area Networks Part 16: Air Interface for Fixed Broadband Wireless Access Systems, 2004.

[9]. Air Interface for fixed broadband wireless access systems, Oct. 2004.

[10]. M. Gerla, R. Bagrodia, L. Zhang, K. Tang, and L. Wang, "TCP over Wireless Multihop Protocols: Simulation and Experiments," IEEE ICC 1999

[11]. M. Gerla, K. Tang, and R. Bagrodia, "TCP Performance in Wireless Multihop Networks," IEEE WMCSA 1999

[12]. V. Bharghavan, "Performance Analysis of a Medium Access Protocol for Wireless Packet Networks," IEEE Performance and Dependability Symposium 1998.

[13]. H. Balakrishnan, V. Padmanabhan, and R. Katz, "The effects of asymmetry on TCP performance," in Proc. 3rd ACM/IEEE Intl. Conference MOBICOM, 1997.

[14]. K. Fall, S. Floyd "Simulation Based Comparison of Tahoe, Reno and Sack TCP”.

\section{BIOGRAPHIES}

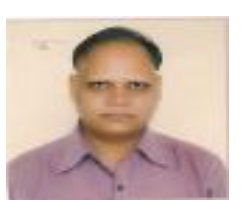

Kailash Chandra Bandhu

Department of Computer Science \& Engineering, Ph.D. Scholar Bhagwant University Ajmer, India

Kailash_bandhu@yahoo.co.in

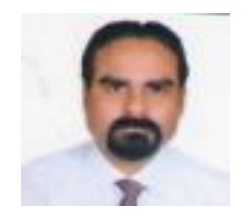

Dr. Rajeev G. Vishwakarma

Department of Computer Science \& Engineering, Shri Vaishnav Institute of Technology and Science

Indore, India

Rajeev@mail.com 